\title{
A Prospective Observational Study on Drug Utilization Pattern and Relation of Alcohol Consumption in Patients with Alcoholic Liver Disease in a Tertiary Care Hospital
}

\author{
Ganesh Nayak*, Cyril Tom, Ditty Molukalayil Skaria, Nehla Yasmeen, Fathima Hasbin CK \\ Department of Pharmacy Practice, Shree Devi College of Pharmacy Mangalore, Airport Road, Kenjar, Dakshina, Karnataka, INDIA
}

\begin{abstract}
Alcoholic Liver Disease is a result of excessive consumption of alcohol and alcohol containing beverages that damages the liver, leading to a buildup of fats, inflammation, and scarring. Objectives: The main aim of our study is an assessment of drug utilization patterns and the relation of alcohol consumption in ALD patients. Methods: A prospective type of observational study in a 700 bedded multispecialty hospital for 6 months. SPSS (Statistical Package for Social Science) version 20. [IBM SPASS statistics (IBM corp. Armonk, NY, USA released 2011)] was used to perform the statistical analysis. Drug therapy and amount, duration and binge of alcohol consumption were assessed. Results: Majority of them consuming alcohol to a greater amount for $10-20$ years. A total of 588 drugs were prescribed to 65 patients. Out of which most commonly prescribed class of drug was proton pump inhibitors, followed by vitamins, antibiotics, and diuretics. The commonly prescribed vitamins, antibiotics and diuretics, hepatoprotective agent were, Vit K(44.6\%), Rifagut (rifaximin)(58.5\%) and spironolactone(50.8\%), pentoxyfyllin $(4.6 \%)$ respectively. Conclusion: The main causes of the patient's lower quality of life are excessive alcohol consumption and noncompliance with the prescribed treatment. All of these factors will exacerbate the disease. To improve their condition and control ALD, patients should abstain from alcohol and strictly adhere to their medication regimen. The patient should be educated enough about the disease and the adherence to medication so that they might be able to upgrade their status of life and be disease free.
\end{abstract}

Key words: Alcoholic liver disease, Fatty liver, Cirrhosis, Alcohol, Drug utilization, Evaluation.

\section{INTRODUCTION}

Prolonged excessive consumption of alcohol was the key germ for developing alcoholic liver disease. The pathologic features of alcoholic liver disease consist of three major injuries, and these injuries are progressive and rarely occur as in pure form. Three major injuries were fatty liver, alcoholic hepatitis, and cirrhosis. ${ }^{1}$

World Health Organization on its modern report specifies that 3.3 million deaths $(6 \%$ of all global deaths) are owed to alcohol use. $^{2}$
Risk factors include the amount of alcoholIn men, fatty liver was produced by $40-80 \mathrm{~g} / \mathrm{d}$ of ethanol use; $160 \mathrm{~g} / \mathrm{d}$ for 10-20 years causes hepatitis or cirrhosis. Only a few people of alcoholics develop alcoholic liver disease i.e 15\% of alcoholics.

Gender-Women reveal increased vulnerability to alcoholic liver disease at amounts $>20 \mathrm{~g} / \mathrm{d}$; two drinks per day is probably secured.

Genetics-Alcoholic cirrhosis has been known to be associated with a protein
DOI: 10.5530/ijopp.15.1.4

Address for correspondence: Mr. Ganesh Nayak, 5th Pharm-D, Department of Pharmacy Practice, Shree Devi College of Pharmacy Mangalore, Airport road, Kenjar, Dakshina-575142, Karnataka, INDIA.

Email id: ganeshnayakhdk@ gmail.com

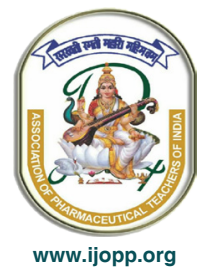


called Patatin-like phospholipase domain-containing protein 3 (PNPLA3), Alcohol injury does not require malnutrition, but obesity and non-alcoholic fatty liver are risk factors. Patients must pay proper attention to nutritional support.

Infection- HCV infection concurrent with alcoholic liver disease is associated with a younger age for severity, more advanced histology, and decreased survival. ${ }^{3}$ Alcohol (ethanol) is readily absorbed from the stomach, but most are absorbed from the small intestine. Alcohol cannot be stored. A small amount of alcohol is being degraded by the gastric mucosa, but a majority of alcohol catabolization occurs mainly in the liver, primarily by alcohol dehydrogenase (ADH). Some portions of it are also catabolized by cytochrome P-450 2E1 (CYP2E1) and the microsomal enzyme oxidation system (MEOS).

Metabolism occurring through the ADH pathway involves the following:

$\mathrm{ADH}$, a cytoplasmic enzyme, oxidizes alcohol into acetaldehyde. Genetic polymorphisms in $\mathrm{ADH}$ cause individual variation in blood alcohol levels i.e an individual consuming the same amount of alcohol but susceptibility to alcoholic liver disease may vary compared to others.

- Acetaldehyde dehydrogenase (ALDH), a mitochondrial enzyme, then oxidizes acetaldehyde into acetate. Chronic alcohol consumption enhances acetate formation. ALDH, which presents at lower levels in the Asian population is more at risk of toxic acetaldehyde effects (eg, flushing); which are similar to those effects of disulfiram, which is due to inhibition of ALDH.

- These oxidative reactions generate hydrogen, which converts nicotinamide-adenine dinucleotide (NAD) to its reduced form (NADH), increasing the redox potential (NADH/NAD) in the liver.

- The increased redox potential inhibits fatty acid oxidation and gluconeogenesis, promoting fat accumulation in the liver.

Chronic alcoholism will cause an increase in the activity of MEOS (mainly at the endoplasmic reticulum site). The main enzyme involved is CYP2E1. When induced, it accounts for $20 \%$ of alcohol biotransformation through the MEOS pathway. As a result, this pathway of reactive oxygen species is generated, leads to oxidative stress and the formation of harmful oxygen-free radicals.

\section{Management}

The first therapeutic goal of patient management in patients with ALD is to induce alcohol withdrawal. The maintenance and supplementation of fluid, calories, vitamins, and minerals are usually shown better patient treatment outcomes.

Abstinence from alcohol- this includes rehabilitation with a multidisciplinary approach. Malnourishment occurs in ALD patients for several reasons including malabsorption, the induction of a catabolic state, the displacement of calories with alcohol. Glucocorticoids, Anti-tumor necrosis factors, antioxidants were the treatment option for ALD. Liver transplantation has been done in case of treatment failure or patient unresponsive to the Therapy or liver damage. ${ }^{4}$

Drug Utilization Evaluation (DUE) is defined as "an ongoing, authorized and systematic process, which is designed to review drug use or prescribing patterns, provide feedback of results to clinicians and other relevant groups, develop criteria and standards which describe optimal use of drug and promote appropriate drug use through education and other interventions". Drug utilization is a system of ongoing and systematic criteria based on the assessment of drug use that helps to ensure drugs are used properly. By assessing drug utilization, it collects, analyses, and interprets drug usage patterns to improve the quality of drug use and patient outcomes. DUE reviews the prescribing patterns and drug use. It also provides feedback on results to develop criteria and standards to promote appropriate drug usage. ${ }^{5}$

\section{MATERIALS AND METHODS}

A prospective observational study was carried out in the Inpatients of the concerned Department for six months at Yenepoya Medical College and Hospital, Mangalore. A patient information sheet was given to the patients and informed consent was obtained from the patient and or caregivers. About 65 patients were selected for the study based on inclusion and exclusion criteria. Patient data were collected using a patient data collection form which included the demographic details, laboratory investigations, diagnosis and medications.

\section{Study Site}

The study was conducted at the concerned department, Yenepoya Medical College and Hospital, Mangalore. It is a 700 bedded Multi-Specialty Tertiary Care Teaching Hospital.

Indian Journal of Pharmacy Practice, Vol 15, Issue 1, Jan-Mar, 2022 
Study Design: This is a Prospective Observational Study.

Study Period: Six months in Yenepoya Medical College and Hospital Mangalore.

\section{Study criteria}

The study was carried out based on the criteria such as patients who are above 18 years of age and are willing to or gives their consent to participate in the study. Patients from both inpatient and outpatient departments were included in our study.

Study participants: below 18years of age, intensive care unit, and pregnant or lactating women were excluded from our study.

Ethical Approval: The study was approved by Yenepoya Ethics Committee 2

Approval date: 16-04-2021 Protocol no: YEC2/757

\section{Data collecting method}

Data will be collected through a review of case sheets in the inpatients of the concerned department. The data collection includes patient details like age, sex, weight, height, history, diagnosis and treatment given to the patients.

\section{Statistical Analysis}

SPSS (Statistical Package for Social Science) version 20. [IBM SPASS statistics (IBM corp. Armonk, NY, USA released 2011)] was used to perform the statistical analysis. Data was entered in the excel spreadsheet. Descriptive statistics of the explanatory and outcome variables were calculated by mean, standard deviation, frequency, and charts.

\section{RESULTS}

\section{Consort flow chart}

Out of 65 patients, the majority of them i.e, 24 $(36.9 \%)$ patients were found in the age group of $40-50$ years followed by $22(33.8 \%)$ patients in the age group of 30-40 years, $11(16.9 \%)$ patients in the age group of 50-60 years, $5(7.7 \%)$ patients in the age group of $20-30$ years, $2(3.1 \%)$ patients in the age group of $60-70$ years and $1(1.5 \%)$ patient in the age group of 70-80 years which is represented in Table 1 .

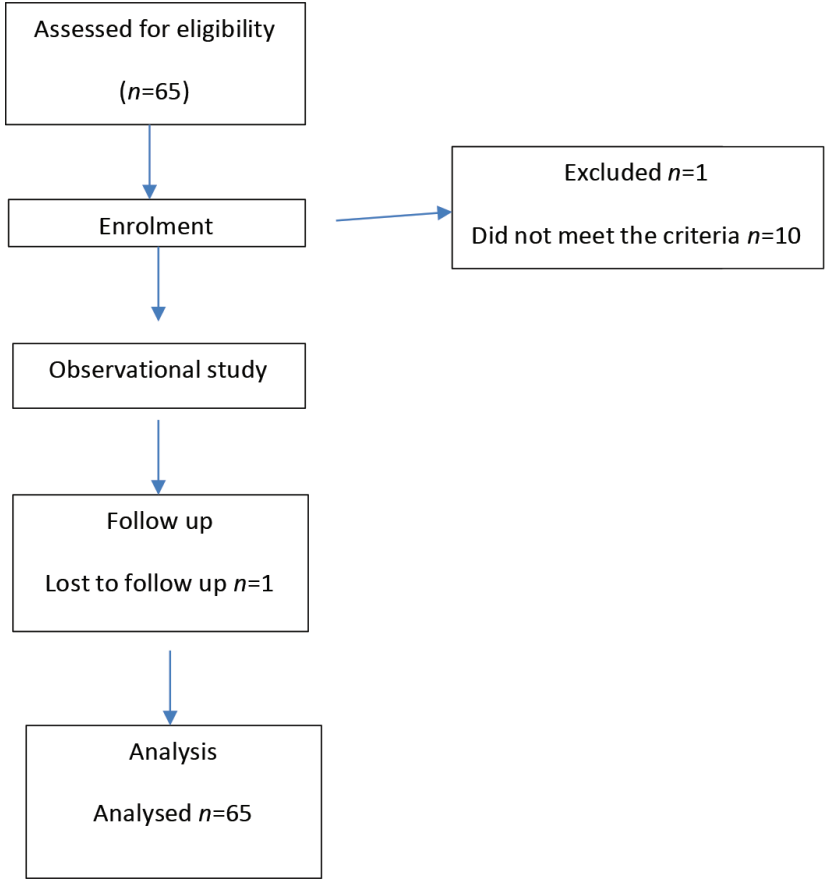

Table 1: Age-wise distribution of study participants.

\begin{tabular}{ccc}
\hline Age (in years) & Frequency & Percent \\
\hline $20-30$ & 5 & 7.7 \\
$31-40$ & 22 & 33.8 \\
$41-50$ & 24 & 36.9 \\
$51-60$ & 11 & 16.9 \\
$61-70$ & 2 & 3.1 \\
$71-80$ & 1 & 1.5 \\
Total & 65 & 100.0 \\
\hline
\end{tabular}

The majority of the study participants were in the age group of 40-50 years.

Table 2: Gender-wise distribution of study participants.

\begin{tabular}{ccc} 
& Frequency & percentage \\
\hline Male & 64 & 98.46 \\
Female & 1 & 1.54 \\
total & 65 & 100 \\
\hline
\end{tabular}

\section{Gender wise distribution of subjects}

Out of 65 subjects, $64(98.46 \%)$ patients were male and $1(1.54 \%)$ were female which is represented by Table 2.

\section{Distribution of subjects based on the duration of alcohol use}

Out of 65 patients, the majority 32(49.2\%) had been consuming alcohol since 10-20 years, $26(40 \%)$ patients were consuming alcohol for less than 10 years which is represented in Figure 5.3. 


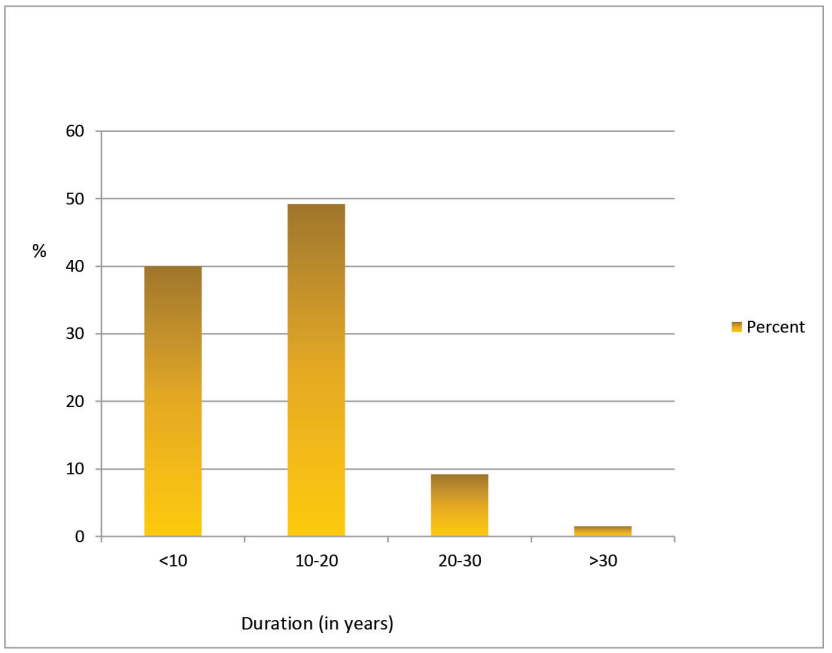

Figure 5.3: Representation of the duration of alcohol consumption.

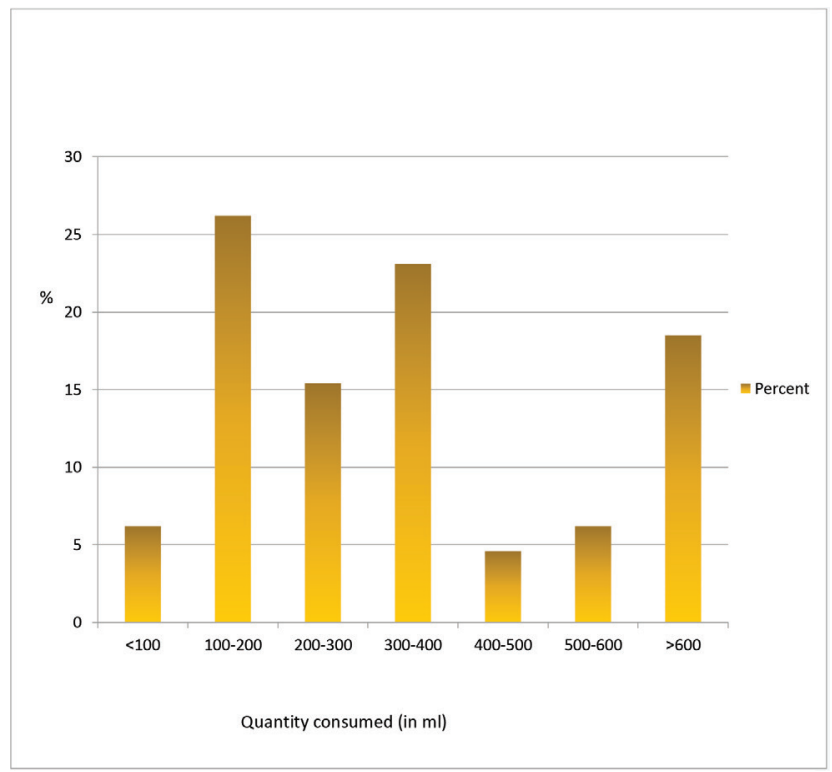

Figure 5.4: Representing the quantity of alcohol consumed. Presence of comorbidities among the alcoholic liver disease patients.

\section{Distribution of subjects based on the quantity of alcohol consumption and age group}

It is observed that $17(26.2 \%)$ patients were consuming $100-200 \mathrm{ml}$ ofalcohol,15(23.1\%) patients were consuming $300-400 \mathrm{ml}$ of alcohol, $12(18.5 \%)$ patients were consumed more than $600 \mathrm{ml}$ of alcohol, (15.4\%) patients were consuming $200-300 \mathrm{ml}$ of alcohol which is represented by Figure 5.4 .

Out of 65 patients, $6(9.2 \%)$ patients have diabetes mellitus, $5(7.7 \%)$ patients have a combination of diabetes and hypertension, 4(6.2\%) patients have comorbidities

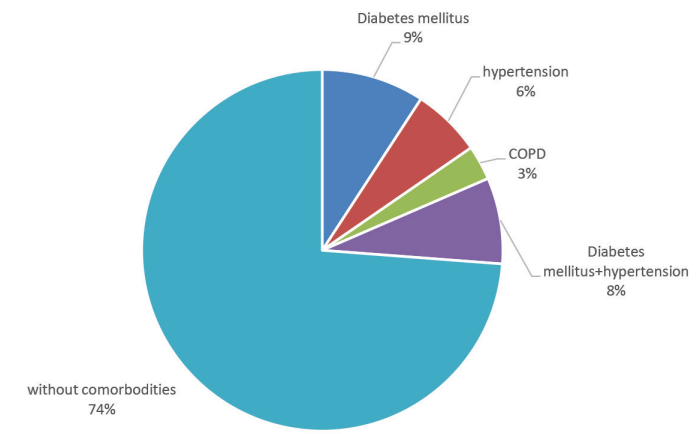

Figure 5.5: Representing the comorbidities among the study participants.

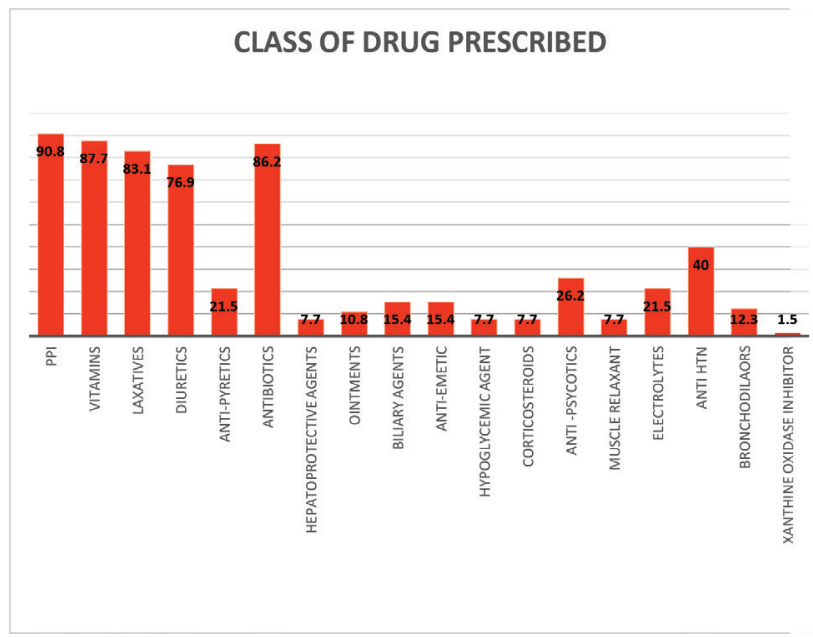

Figure 5.6: Representing the class of drug prescribed.

hypertension and 2(3.1\%) patients have COPD along with the alcoholic liver disease. The majority i.e, $48(73.8 \%)$ patients were not having any comorbidities which are represented by Figure 5.5.

\section{Distribution of drugs based on the class of drug}

Among 65 patients, laxatives were the main therapeutic agent for the patients. A total of 588 drugs were prescribed to 65 patients. The most prescribed class of drug was PPI (90.8\%) followed by Vitamins (87.7\%) and antibiotics $(86.2 \%)$ laxative $(83.1 \%)$, diuretics (76.9\%) anti-hypertensive (40\%) Anti-psychotics (26.2\%) Antipyretic and electrolytes (21.5\%) each Biliary agents and antiemetics (15.4\%) each bronchodilator (12.3\%) Hepatoprotective agents, Muscle relaxant, Corticosteroids, Hypoglycaemic agents (7.7\%) each, Indian Journal of Pharmacy Practice, Vol 15, Issue 1, Jan-Mar, 2022 
Xanthine Oxidase Inhibitors class of drug was least prescribed (1.5\%) which is represented by Figure 5.6.

Pantoprazole were prescribed to 57 patients (87.7\%), lactulose were prescribed to 54 patients (83.1\%), thiamine were prescribed to 49 patients (75.4\%), Rifagut were prescribed to 38 patients $(58.5 \%)$, spironolactone were prescribed to 33 patients $(50.8 \%)$, furosemide were prescribed to 32 patients (49.2\%), Vitamin $\mathrm{K}$ and Antibiotics were prescribed to 29 patients (44.6\%) each were prescribed to 28 patients(43.1\%), Taxim were prescribed to 23 patients (35.4\%), Inderal were prescribed to 20 patients $(30.8 \%)$, Folic acid were prescribed to

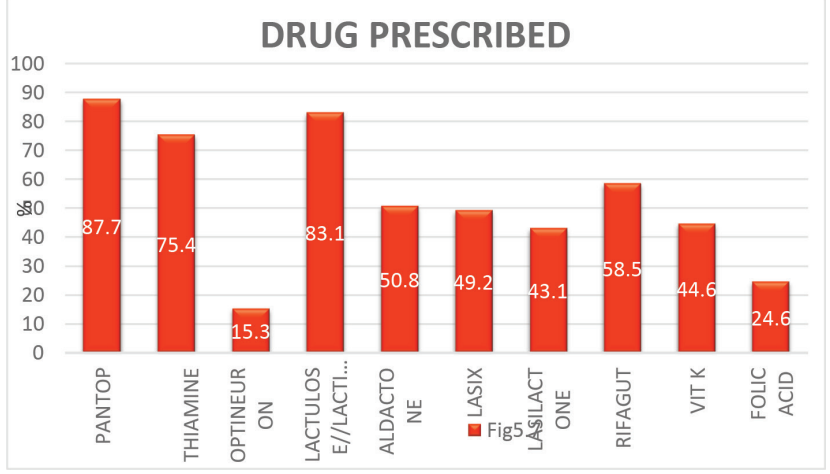

Figure 5.7: Representation of drug prescribed.

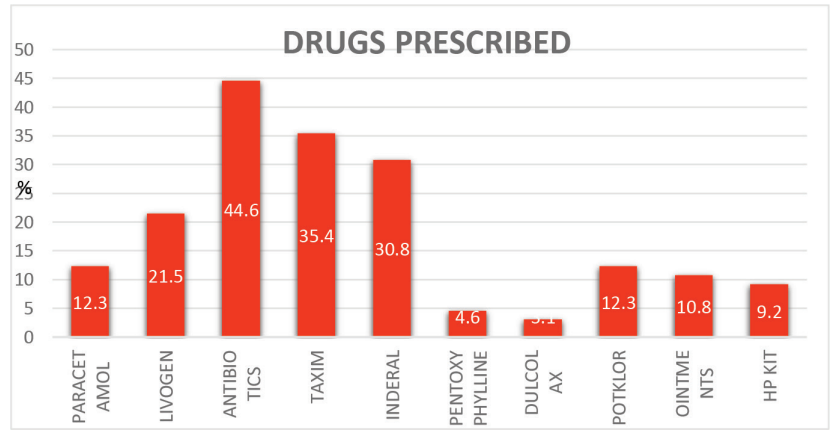

Figure 5.8: Representation of drug prescribed.

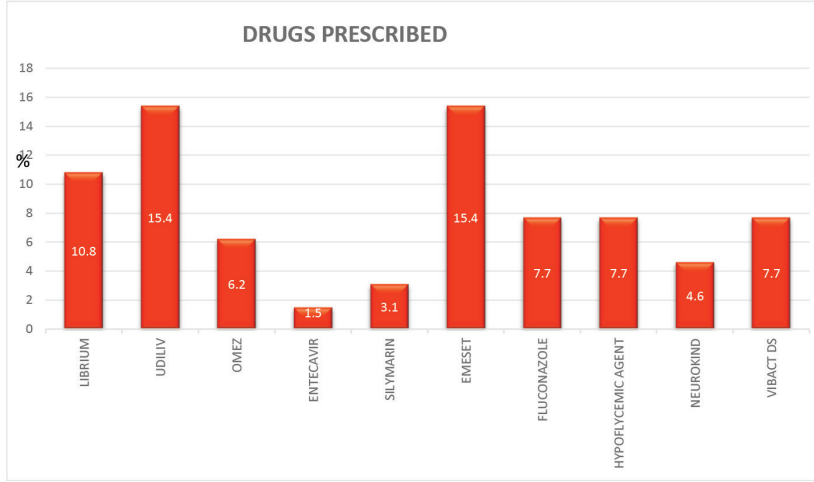

Figure 5.9: Representation of drug prescribed.

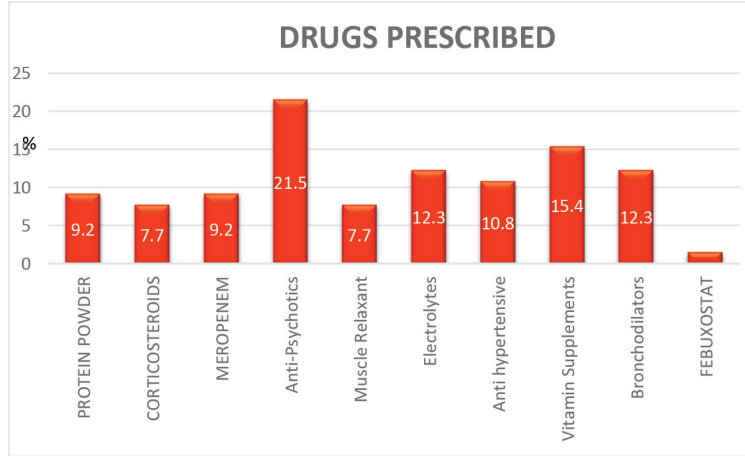

Figure 5.10: Representation of drug prescribed.

16 patients $(24.6 \%)$, livogen were prescribed to 14 patients (21.5\%), Anti-psychotics were prescribed to 14 patients (21.5\%), Optineuron, Udiliv, emeset, Vitamin supplements were prescribed to 10 patients (15.4\%) each, Potklor, paracetamol, electrolytes, bronchodilators were prescribed to 8 patients (12.3\%) each, Ointments, Librium, Anti-Hypertensive were prescribed to 7 patients $(10.8 \%)$ each, HP kit, protein powder, meropenem were prescribed to 6 patients $(9.2 \%)$ each, Fluconazole, Hypoglycaemic agents, Vibact DS, muscle relaxant were prescribed to 5 patients (7.7\%)each, Omez were prescribed to 4 patients (6.2\%), Pentoxyphylline, neurokind were prescribed to 3 patients (4.6\%) each, Dulcolax, silymarin were prescribed to 2 patients (3.1\%) each, Entecavir, febuxostat were prescribed to 1 patient $(1.5 \%)$ each which is represented by Figure 5.7, 5.8, 5.9, 5.10 .

\section{DISCUSSION}

The study was carried out to analyze which is the most commonly prescribed drug for alcoholic liver disease, the amount and duration of alcohol consumed by a patient with this disease in a tertiary care hospital.

Limitations of this study are therapeutic outcome was evaluated based on symptomatic relief. Alcoholic liver disease patients with psychiatric disorders, ICU patients were excluded. This study was conducted only in one hospital and therefore it included only patients from the hospital for a short period of 6 months. Hence it cannot be generalized to the population of India having the disease.

The future perspective of our study was evaluation of alcohol consumption can give you an economic burden on society. Pharmacoeconomic evaluation studies can be done for assessment of ALD therapy. Outcome of the therapy can be clinically evaluated by analyzing the blood samples and liver function tests. Multicenter 
study involving a larger number of patients and for a longer duration of time can give more accurate results.

Distribution of drugs were studied among the 65 patients and it was found that Pantoprazole were prescribed to 57 patients (87.7\%), Lactulose were prescribed to 54 patients (83.1\%), Thiamine were prescribed to 49 patients (75.4\%), Rifagut was prescribed to 38 patients (58.5\%), Spironolactone were prescribed to 33 patients $(50.8 \%)$, Furosemide were prescribed to 32 patients (49.2\%), Vitamin K and Antibiotics were prescribed to 29 patients $(44.6 \%)$ each, Lasilactone were prescribed to 28 patients (43.1\%), Taxim were prescribed to 23 patients(35.4\%), Inderal was prescribed to 20 patients $(30.8 \%)$, Folic acid were prescribed to 16 patients $(24.6 \%)$, livogen were prescribed to 14 patients (21.5\%), Anti-psychotics was prescribed to 14 patients (21.5\%), Optineuron, Udiliv, Emeset, Vitamin supplements were prescribed to 10 patients $(15.4 \%)$ each, Potklor, Paracetamol, Electrolytes, Bronchodilators were prescribed to 8 patients (12.3\%) each, Ointments, Librium, AntiHypertensive were prescribed to 7 patients(10.8\%) each, HP kit, protein powder, meropenem were prescribed to 6 patients $(9.2 \%)$ each, Fluconazole, hypoglycemic agents, Vibact DS, muscle relaxant were prescribed to 5 patients(7.7\%)each, Omez were prescribed to 4 patients (6.2\%), Pentoxyphylline, neurokind were prescribed to 3 patients (4.6\%) each, Dulcolax, silymarin were prescribed to 2 patients $(3.1 \%)$ each, Entecavir, Febuxostat were prescribed to 1 patient $(1.5 \%)$ each. The most commonly prescribed drug was found to be Pantoprazole and Lactulose while the least commonly prescribed was Entecavir and Febuxostat. Thus, is supported by Zohreh Eftekhari et al. ${ }^{6}$

Out of the 65 patients, the majority of patients were consuming alcohol for 10-20 years that is $32(49.2 \%)$ followed by less than 10 years 26(40\%), 20-30 years $6(9.2 \%)$, less than 30 years $1(1.5 \%)$ which is supported by V. T. Savolainen et al.

Out of the 65 patients, it was found that 17(26.2\%) patients were consuming $100-200 \mathrm{ml}$ of alcohol,15(23.1\%) patients were consuming $300-400 \mathrm{ml}$ of alcohol, $12(18.5 \%)$ patients were consumed more than $600 \mathrm{ml}$ of alcohol,10(15.4\%) patients were consuming $200-300 \mathrm{ml}$ of alcohol. The majority of the patients are those consuming $100-200 \mathrm{ml}$ of alcohol which was supported by V. T. Savolainen et al. ${ }^{7}$

The distribution of a class of drugs was studied among the 65 patients and it was found that laxatives were the main therapeutic agent for the patients. A total of 588 drugs were prescribed to 65 patients. Most prescribed class of drug was PPI (90.8\%) followed by Vitamins (87.7\%) and antibiotics (86.2\%), laxative (83.1\%), diuretics (76.9\%), anti-hypertensive (40\%) anti-psychotics (26.2\%), anti-pyretic and electrolytes $(21.5 \%)$ each, biliary agents and anti-emetics (15.4\%) each, bronchodilators (12.3\%), hepatoprotective agents, muscle relaxant, corticosteroids, hypoglycemic agents (7.7\%) each, xanthine oxidase inhibitors class of drug was least prescribed (1.5\%). This study was supported by Zeebaish $S$ et al. ${ }^{8}$

Out of 65 patients, 64 patients were males (98.46\%) and 1 patient was female (1.54\%). This was supported by A.M. Patil et al. ${ }^{9}$

The study participants were assessed for comorbidities and was found that out of the 65 patients, $48(73.8 \%)$ patients were not having any comorbidities, $6(9.2 \%)$ patients have diabetes mellitus, $5(7.7 \%)$ patients have a combination of diabetes and hypertension, $4(6.2 \%)$ patients have hypertension and 2(3.1\%) patients have COPD along with the alcoholic liver disease. The majority of our study population had no comorbidities. This is supported by Evan J. Raff et al. ${ }^{10}$

The patients were classified into different age groups, that is $20-30,31-40,41-50,51-60,61-70,71-80$ and the number of patients in each group was $5(7.7 \%)$, 22(33.8\%), 24(36.9\%), 11(16.9\%), 2(3.1\%), 1(1.5\%) respectively. The majority of alcoholic liver disease patients were between 40-50 and the least number of patients was in the age group 70-80 which is supported by the study conducted by S.M. Biradar $e t$ al. ${ }^{11}$

\section{CONCLUSION}

Excessive alcohol consumption and non-compliance to the treatment given are the main reasons for the decreased quality of life of the patients. All these will lead to the worsening of the disease. Alcohol consumption leads to an economic burden on society. Abstinence from alcohol and complete adherence to medication should be strictly followed by the patients to improve their condition and control ALD. The patient should be educated enough about the disease and the adherence to medication so that they might be able to improve their quality of life and be disease-free.

\section{ACKNOWLEDGEMENT}

The authors of the study are heartily thankful for the guidance and support received from all the members who contributed to this project, it was vital for the 
success of the project.

\section{Funding}

This research work doesn't receive a specific grant from any funding agency in the public.

\section{CONFLICT OF INTEREST}

The authors declare no conflict of interest.

\section{ABBREVIATIONS}

ALD: Alcoholic liver disease; DUE: Drug utilization and evaluation; PNPLA3: Patatin like phospholipase domain-containing protein 3; ADH: Alcohol dehydrogenase; CYP2E1: Cytochrome P-450 2E1; MEOS: microsomal enzyme oxidation system; ALDH: Acetaldehyde dehydrogenase; NAD: nicotinamide adenine dinucleotide.

\section{REFERENCES}

1. Jameson JL, Fauci AS, Kasper DL, Hauser SL, Longo DL. Joseph Loscalzo Harrison's principles of internal medicine; $20^{\text {th }}$ edition [2018]; chapter-335
2. Ashwani SKMD, Bataller MSFACG1, Ramon MD, Joseph AMD, FACG MS. (GRADE Methodologist)3; Kamath, Patrick S MD4; Shah, Vijay H MD, FACG4 ACG Clinical Guideline: Alcoholic Liver Disease, American Journal of Gastroenterology. 2018 ;113(2):175-94. DOI: 10.1038/ajg.2017.469

3. Nicholas T, Orfanidis MD. Thomas Jefferson University Hospital Last full review/ revision. Content last modified Jul 2019 MSD PROFESSIONAL MANUAL. 2019

4. Ferenci P, Dragosics B, Dittrich H, Frank H, Benda L, Lochs H, et al. Randomised controlled trial of silymarin treatment in patients with cirrhosis of the liver. $\mathrm{J}$ Hepatol.1989;9:105-113.

5. Parthasarathi G, Nyfort-Hansen K, Nahata MC, The textbook of Clinical Pharmacy Practice, Second edition. 2012;447-64.

6. Eftekhari Z, et al. Drug utilization pattern of patients with alcoholic liver disease in Jayanagar General Hospital, Bangalore, India. Archives of Pharmacy Practice. 2020;11( S1).

7. Savolainen VT et al. Alcohol consumption, and alcoholic liver disease: evidence of a threshold level of effects of ethanol. Alcoholism: Clinical and Experimental Research. 1993;17(5):1112-7.

8. Zeebaish S, et al. A prospective observational study on prescribing patterns of drugs used in alcoholic liver disease patients at a tertiary care teaching hospital. International Journal of Basic and Clinical Pharmacology. 2017;6(6):1386-92.

9. Patil AM, Arifulla M, Yendigeri SM, Sajanar BB. Study of Alcoholic Liver Cirrhosis in Hospital-Based Patients, Bijapur, Northern Karnataka, India. International Journal of Current Medical and Applied Sciences. 2015;7(1):16-20.

10. Raff EJ, Kakati D, Bloomer JR, Shoreibah M, Rasheed K, Singal AK. Diabetes mellitus predicts the occurrence of cirrhosis and hepatocellular cancer in the alcoholic liver and non-alcoholic fatty liver diseases. Journal of clinical and Translational Hepatology. 2015;3(1):9.

11. Biradar SM, Gelada D, Mounika MV, Meghana P, Bharathi M, Ambali AP, et al. Assessment of clinical profile and treatment chart review for alcoholic liver disease (ald) patients: a prospective and observational study. Journal of Drug Delivery and Therapeutics. 2018;8(5):437-441. 